\title{
Research Progress on Numerical Simulation of Two-phase Flow in the Gas-solid Fluidized Bed
}

\author{
Shujie Sun, Xiaosai Dong, Jie Wang, Haodong Zhang and Zhenya Duan* \\ College of Electromechanical Engineering, Qingdao University of Science and Technology, Qingdao 266061, Shandong, China
}

\begin{abstract}
It is difficult to accurately measure the parameters of solid particles in the experiment of the gas-solid fluidized bed. The numerical simulation plays an important role to accurately describe flow characteristics in the fluidized bed. Combined with the research work of the research group, this paper analyzes the application of numerical simulation of fluidized bed from the aspects of gas-solid coupling algorithm, drag model, flow characteristics, and reaction characteristics based on the previous studies. The specificity improvement of the gas-solid coupling algorithm and the regional application of the drag model is the trend of the recent development of numerical simulation. Previous studies mainly focus on the gas-solid two-phase flow field characteristics in the traditional fluidized bed, but few on the complex flow characteristics such as gas-solid reverse flow and the coupling with reaction characteristics. It is of great significance for designing a novel fluidized bed reactor to realize gas-solid continuous reaction to establish and improve the numerical simulation method of gas-solid non-catalytic reaction.
\end{abstract}

\section{Introduction}

Gas-solid fluidized bed has been widely used in petrochemical, pharmaceutical, drying, and other fields, the study of gas-solid two-phase flow has important significance for industrial equipment design and optimization of operating conditions. The current research on gas-solid fluidized bed is mainly carried out by experimental studies and numerical simulations: most of these experimental methods are based on experimental setups, which are expensive and involve considerable engineering effort, and very few studies are conducted on industrial setups; numerical simulations allow more accurate calculation, prediction and study of gas-solid two-phase flow, and more intuitive access to the processes and details of gas-solid flow at the particle and mesh scales, which are difficult to measure experimentally, resulting in a wealth of microscopic information. The prediction results can be helpful for the development of novel reaction equipment and the optimization of internals, however, the reasonableness of the parameters and the reliability of the prediction results are still difficult to be verified theoretically and largely depend on experimental data for examination and determination. As an important method complementary to experimental research, numerical simulation will play a more important role in the analysis and research process of gas-solid fluidized bed.

The particles are often used as catalysts in the traditional fluidized bed, which properties do not change in the reaction process, and are easy to be simplified in numerical simulation, so that the simulation results match well with the actual flow conditions. However, there are numerous gas-solid non-catalytic reactions in pharmaceutical and chemical production, that is, solid particles react directly with the gas without catalyzing the reaction. For example, the carboxylation reaction of sodium phenolate and $\mathrm{CO}_{2}$ in the production process of salicylic acid, it is usually produced using the agitator which is the batch production equipment. If the fluidized bed technology can be used to achieve the continuous operation of this reaction, the product quality and output would be improved significantly. The research group has carried out an in-depth study on the process conditions, reaction thermodynamics, reaction mechanism and reaction kinetics of sodium phenolate carboxylation, and proposed a novel fluidized bed [1] that can realize the efficient contact between gas and solid particles and continuous reaction to generate solid particle products. The reactor has a big difference with the traditional fluidized bed in terms of feeding mode and internals settings. Sodium phenolate particles fall overcoming the drag between the gas-solid phase and then evenly distributed in the bed through the role of the internals. $\mathrm{CO}_{2}$ gas is both fluidizing medium and involved in reactions. It mainly solves the technical problems of solid particles react with gas to generate solid particles, effectively ensure full contact between the gas-solid phase, reduce the axial back mixing of solid particles, and improve the reaction efficiency.

In recent years, the improvement of simulation accuracy mainly depends on modification of drag model and coupling algorithm, the research on simulation of gas-solid fluidized bed mainly focuses on gas-solid flow characteristics, but the mass transfer and reaction characteristics are mentioned in some literature. This study summarizes the progress of numerical simulation technology in gas-solid flow, discusses

\footnotetext{
Corresponding author: qust_zyduan@163.com
} 
the simulation methods and results of the coupling with reaction characteristics in gas-solid fluidized bed and analyzes its future development direction, and it is of great significance to the application of numerical simulation in the field of the fluidized bed and the related research, provide useful help for the exploration of simulation that can reasonably describe the reaction characteristics of gas-solid non-catalytic reactions.

\section{Drag model and coupling algorithm}

The gas-solid coupling algorithm can be divided into Two-Fluid model (TFM) and Discrete phase model (DPM). The selection of the coupling algorithm depends not only on whether the gas-solid interaction is considered, but also on the particle concentration. Generally, when the particle concentration is low, to simplify the algorithm and speed up the operation, the DPM model can be selected[2-6]. In this model, the gas is regarded as a continuous phase, and the Stokes equation is solved in the framework of Euler, particles are regarded as discrete phases and the orbit equation is solved in the Lagrangian framework. The interaction between particles is ignored in the DPM model, but it can not be ignored in dense gas-solid fluidized bed. In this case, the dense discrete phase model (DDPM) can be selected[7], which is an extension of the traditional DPM model. When the particle concentration is high or the flow characteristics of gas-solid two-phase need to be studied accurately, the TFM model is often used[8-22]. In this model, both gas and solid phases are regarded as continuous media, and the interaction between particles is calculated by Kinetic theory of granular flow (KTFG) and particle temperature transport equation. Among them, the Stokes equation is solved in the Eulerian framework for the gas phase, and the conservation equation is solved for the particle phase in the Eulerian framework.

In addition to the traditional TFM model, many coupling algorithms[2, 7, 23-28] have been proposed to simulate dense gas-solid two-phase flow effectively, most of these algorithms are aimed at specific working conditions, and have the characteristics of large limitations and single application range, so they are difficult to be popularized. In short, in the face of more complex simulation requirements, gas-solid coupling algorithm is more and more difficult to achieve a wide range of applications, accurate calculation and save computing resources and other aspects of comprehensive consideration, the improvement of its specificity is a new trend in the development of current numerical simulation.

Bian et al. [29] compared the results obtained by the TFM model, the DPM model, and experiment on gas-solid flow in a dense fluidized bed, and the results indicated that the drag force between the two phases was the key to the accuracy of the simulation. The drag force is the main force in the vertical flow of gas-solid two phases and is an important parameter to characterize the interaction and momentum exchange between gas-solid two phases. The correct drag model and coefficient of drag force are the key to describe gas-solid two-phase motion accurately in numerical simulation[30-31]. Studies have shown that the Gidaspow model can more accurately simulate the fluidization and flow characteristics of large particles in a gas-solid fluidized bed. On the contrary, the Stokes-Cunningham drag law is more suitable for characterizing the flow behavior of small-diameter particles [10]. However, existing models of drag force are difficult to accurately describe the increasingly complex industrial production situation, many scholars have integrated and improved the existing drag force model [8-9, 32] and achieved good results. Some scholars also proposed a gas-solid drag force model based on bubbles or particle clusters [14, 33-34]. These models can accurately represent the gas-solid phase drag force in a specific region under a specific working condition, but their application scope is relatively narrow. It is difficult for these models to fully reflect the characteristics of gas-solid flow in the case of a complex structure in fluidized bed equipment or a large difference in gas-solid flow in different areas of the same equipment. Therefore, scholars have carried out a lot of work on the research of using the drag model in stages[11, 13, 37]. Segmental use of the drag force model can effectively predict the transient particle distribution in different regions observed in the experiment. It also shows that the application of the drag model in numerical simulation is more and more regional and specific.

\section{Gas-solid flow characteristics}

The simulation of gas-solid two-phase flow mainly involves fluidized bed, spouted bed, and coal-fired boiler. It is difficult to directly observe the gas-solid two-phase flow characteristics in the equipment under the experimental state. Therefore, it has gradually become the mainstream to investigate the gas-solid two-phase flow characteristics by means of numerical simulation. The research on the characteristics of gas-solid two-phase flow field is relatively mature, which mainly involves gas-solid mixing [19, 25, 35-38], diffusion [36], aggregation [39-40], separation [20, 41], backmixing [40, 42], uniformity [43-44] and heat transfer [45-47]. However, the particles properties are more complex. The investigation of particle characteristics mainly focuses on the movement law $[17-18,20,30,39,42-43$, 48-53] and the distribution of particles. It is a difficult problem to find a scientific and effective method to visually characterize the flow state of particles in the fluidized bed.

The structure of the fluidized bed and the installation of internals can significantly improve the flow state and mixing effect of gas-solid two-phase. Reasonable selection and design can effectively achieve specific industrial production purposes and improve industrial production efficiency. The geometry size and bed structure will also directly affect the gas-solid flow characteristics and the performance of the fluidized bed [54-61]. Transverse internals such as grid trays will increase the turbulence degree and the residence time of the particles, and increase the reaction efficiency [15, 22, 62-63]. The air distributor is generally located behind the gas inlet, and the gas flowing into the equipment is evenly distributed in the bed through the redistribution of the air distributor, which can effectively improve the mixing efficiency of gas-solid two-phase and enhance the controllability of equipment operation $[55,64]$. The effects of operating conditions such as superficial gas velocity [2-3, 
$16,22,30,39,44,54-55,65-67]$, bed height $[3,66]$ and feed position $[7,20]$ on particle distribution, separation efficiency and residence time in fluidized bed can not be ignored. The application of these variables to numerical simulation can realize the organic combination of numerical simulation and experimental research, and the obtained results can effectively predict the flow field inside the equipment during actual operation. The study of gas-solid two-phase flow in the fluidized bed is of great significance in the design of industrial equipment and the optimization of operating conditions. However, few studies have investigated the reverse flow of gas-solid in fluidized bed or more complex fluid characteristics.

\section{Mass transfer and reaction characteristics}

With the continuous optimization of gas-solid coupling algorithm and drag model, as well as the constant maturity of research on gas-solid two-phase flow field characteristics, CFD has been applied in gas-solid two-phase mass transfer, heat transfer and reaction characteristics gradually. Especially in reaction characteristics, it is often used to predict the gas-solid distribution [40, 68-73], velocity [72-73], temperature [69, 73-75], reaction conversion rate [73, 76-77], reaction efficiency $[6,21,69-70,74]$ in a fluidized bed.

Early scholars mainly studied gas-gas reactions $[6,74$, 68-69, 78-81] and gas-combustion reactions [47, 72, 75, 77] with solid particles as catalysts, solid particles do not directly participate in the reactions, so it is easier to simplify the numerical model. With the increasing demand of the pharmaceutical and chemical industries for continuous production process, the mass transfer and reaction characteristics between gas-solid two phases in a fluidized bed have gradually become a new research direction [39-40, $71,82-84]$, and its research methods can provide a reference for the simulation of gas-solid carboxylation reaction characteristics in a fluidized bed.

\section{Conclusion and outlook}

With the in-depth study of gas-solid two-phase flow in a fluidized bed, especially the increasingly mature CFD technology, the simulation results can help researchers to effectively understand and improve the gas-solid two-phase fluid flow characteristics and to combine with engineering practice. Through the analysis of the article, the research conclusions are as following:

(1) The existing gas-solid coupling algorithm and drag model are difficult to accurately represent the numerical models. Therefore, the specificity improvement of gas-solid coupling algorithm and the regionalized applications of drag model are the recent trends of numerical simulation;

(2) The research on gas-solid flow characteristics in fluidized bed by numerical simulation is relatively mature. However, the investigation on gas-solid reverse flow or complex fluid characteristics is insufficient. In addition, effective methods are needed to quantitatively characterize the flow characteristics of particles;
(3) There are few researches on the coupling of gas-solid flow with reaction characteristics in the gas-solid fluidized bed, but some scholars have begun to carry out this work. In engineering practice, there are many reaction processes in which solids participate in the reaction and generate solid products. It is very important to establish and improve the numerical simulation method which can reflect the influence of reaction characteristics on the flow characteristics, which are of great significance for the development and use of a new fluidized bed reactor to realize gas-solid continuous reaction.

\section{References}

1. Z.Y. Duan, S.P. Li, J.M. Zhang, E.L. Xu, (ZL201720387404, 2017)

2. S.L. Yang (Zhejiang University, Hangzhou, 2014)

3. J.R. Gu, Y.J. Shao, X.J. Liu, W.Q. Zhong, A.B. Yu, Chem. Eng. Sci. 192, 619-633 (2018)

4. C. Yu, F.Q. Si, Y.S. Dong, X. Jiang, J. Southeast Univ. 49, 133-140 (2019)

5. Y.J. Shao, J.R. Gu, W.Q. Zhong, A.B. Yu, Powder Technol. 350, 81-90 (2019)

6. J. Xie, W.Q. Zhong, B.S. Jin, Y.J. Shao, Y.J. Huang, Adv. Powder Technol. 24, 382-392 (2013)

7. A.J. Li, L.Y. Zhu, C. Liu, G.T. Wang, M.Y. Zhang, K. Wang, Z.B. Wang, Powder Technol. 354, 696-708 (2019)

8. S.W. Hu, X.H. Liu, Chem. Eng. Sci. 205, 14-24 (2019)

9. X.P. Qiu, L.M. Wang, N. Yang, CIESC J. 69, 1867-1872 (2018)

10. D.H. Chao, X.Y. Guo, J. Univ. Shanghai Sci. Technol. 32, 333-339 (2010)

11. S.B. Liu, T. Zeng, H.B. Lin, Min. Equip. 43, 99-103 (2015)

12. K. Hong, M.Q. Cao, Y. Xu, X.Y. Gao, S.M. Zhou, X. Gu, Comput. Appl. Chem. 32, 1417-1420 (2015)

13. X. Gao, C. Wu, Y.W. Cheng, L.J. Wang, X. Li, Powder Technol. 228, 1-13 (2012)

14. J. Chang, J.J. Zhao, K. Zhang, J. Gao, Powder Technol. 304,134-142 (2016)

15. J.J. Zhang, F. Wu, X.X. Ma, J. Yang, J. Chem. Eng. Chin. Univ. 31, 776-783 (2017)

16. W. Hassen, M. Dhrioua, L. Kolsi, V. Anbumalar, A. Al-Rashed, M.N. Borjini, Int. J. Mech. Sci. 144, 438-451 (2018)

17. Z.H. Shen, Q.Q. Sun, G.D. Liu, H.L. Lu, Y.L. Ding, J. Eng. Thermophys. 28, 968-970 (2007)

18. C. Wu, X. Gao, Y.W. Cheng, L.J. Wang, X. Li, CIESC J. 64, 858-866 (2013)

19. X.Y. Lan, W.C. Yan, C.M. Xu, J.S. Gao, Z.H. Luo, Powder Technol. 262, 106-123 (2014)

20. A.J. Li, L.Y. Zhu, Z.B. Wang, J. Chem. Eng. Chin. Univ. 32, 1034-1041 (2018)

21. R. Tritippayanon, R. Piemjaiswang, P. Piumsomboon, B. Chalermsinsuwan, Appl. Energy 250, 493-502 (2019) 
22. X.L. Zhang (East China University of Science and Technology, Shanghai, 2018)

23. C.L. Wu, O. Ayeni, A.S. Berrouk, K. Nandakumar, Chem. Eng. Sci. 118, 221-244 (2014)

24. F. Farivar, H. Zhang, Z.F. Tian, A. Gupte, Powder Technol. 360, 1017-1027 (2019)

25. L.B. Ren (Shandong University, Jinan, 2015)

26. M.M. Fang, K. Luo, S.L. Yang, K. Zhang, J.R. Fan, J. Eng. Thermophys. 36, 545-550 (2015)

27. C.Z. Yang, C.M. Chen, G.X. Liu, Y.F. Duan, CIESC J. 67, 2748-2755 (2016)

28. W.D. Yin, Q.G. Wang, J.F. Lv, H.R. Yang, J. China Univ. Min. Technol. 48, 430-436 (2019)

29. W. Bian, X. Chen, J. Wang, Chem. Eng. Sci. 210, 17 (2019)

30. Z.G. Yuan (Harbin Institute of Technology, Harbin, 2015)

31. J.Y. Li (China University of Petroleum, Beijing, 2016)

32. X.Y. Zheng (Nanjing University of Aeronautics and Astronautics, Nanjing, 2016)

33. X.J. Song (Harbin Institute of Technology, Harbin, 2018)

34. X.X. Jiang, D. Li, S.Y. Wang, M. Hassan, W.J. Cai, W.Q. Chen, H.L. Lu, Int. J. Multiphase Flow 122, 103126 (2020)

35. B. Ren, Y.J. Shao, W.Q. Zhong, B.S. Jin, Z.L. Yuan, Y. Lu, Powder Technol. 222, 85-94 (2012)

36. K. Zhang (Zhejiang University, Hangzhou, 2012)

37. L.P. Wei, G.D. Jiang, H.P. Teng, J. Hu, J.B. Zhu, Particuology 49, 95-104 (2020)

38. G.C. Wei (Shenyang Aerospace University, Shenyang, 2017)

39. S.Y. Wang (Harbin Institute of Technology, Harbin, 2008)

40. L. Peng (China University of Petroleum, Beijing, 2017)

41. A. Hashemisohi, L.J. Wang, A. Shahbazi, H. Amini, Particuology 49, 191-204 (2019)

42. P. Lv (China University of Mining and Technology, Xuzhou, 2017)

43. C.L.Yang, J.H. Bai, F. Wu, X. Ma, J. Yang, J. Chem. Eng. Chin. Univ. 33, 1415-1423 (2019)

44. C.X. Wang, J.Z. Zhang, X.Y. Lan, J.S. Gao, J. Zhu, Ind. Eng. Chem. Res. 59, 437-449 (2020)

45. X. Shi (Harbin University of Science and Technology, Harbin, 2019)

46. F.H. Guo, Z.P. Zhong, Waste Biomass Valorization 11, 1115-1123 (2018)

47. L.J. Xu (Zhejiang University, Hangzhou, 2017)

48. B. Luo, H. Dong, H.Z. Li, Proceedings of the 10th national energy and thermal engineering academic annual conference (Energy and Thermal Engineering Branch of China Metal Society, Hangzhou, 2019)

49. X. Zhang (Northwest University, Xian, 2018)
50. M.H. Chen, H.F. Lu, Y. Jin, X.L. Guo, X. Gong, H.F. Liu, Chem. Eng. Res. Des. 155, 1-11 (2020)

51. Q. Chen, H.H. Liu, D.Q. Wang, K. Zeng, J. Bao, P. Chen, H.P. Yang, H.P. Chen, J. China Univ. Min. Technol. 48, 415-421 (2019)

52. M.S. Liu, X.F. Lu, Q.H. Wang, J. Xu, J. Yan, Z. Xu, S.L. Zhou, J.X. Lei, C.X. Liu, X. Xie, Proc. CSEE 39, 543-549 (2019)

53. Y.C. Zhang, W.M. Yi, Z.H. Li, P. Fu, N.N. Wang, C.Y. Tian, Trans. Chin. Soc. Agric. Mach. 50, 281-289 (2019)

54. H. Zhou (The University of Chinese Academy of Sciences, Beijing, 2017)

55. Y. Zhang, Y. Jia, J. Xu, J.W. Wang, C.L. Duan, W. Ge, Y.M. Zhao, Chem. Eng. Process. 148, 107825 (2020)

56. Q.Y. Tu, H.G. Wang, Particuology 49, 205-217 (2019)

57. X. Yao, S.C. Wang, D. Lv, S.X. Zhang, G.S. Du, L.D. Zhang, Nonferrous Metall. Equip. 02, 31-35 (2019)

58. X.J. Hu, S.J. Liu, J. Liu, S.Y. Cheng, Y.N. Xu, F.H. Wang, X.P. Wen, J. Henan Polytech. Univ. Nat. Sci. 39, 94-99 (2020)

59. F. Wu, X.X. Che, Z.Y. Huang, H.J. Duan, X.X. Ma, W.J. Zhou, ACS Omega 5, 1014-1024 (2020)

60. T.Q. Tang (Harbin Institute of Technology, Harbin, 2017)

61. H. Che, Y.X. Zhang, Boiler Technol. 50, 26-32 (2019)

62. S. Yang (University of Chinese Academy of Sciences, Beijing, 2016)

63. W.Q. Liu (Qingdao University of Science and Technology, Qingdao, 2015)

64. Q. Zhou, C.B. Jiang, K.D. Cui, J. Ding, W. Chen, S.Z. Chen, J.J. Guo, China Powder Sci. Technol. 26, 13-19 (2020)

65. S.L. Yang, H. Wang, Y.G. Wei, J.H. Hu, J.W. Chew Powder Technol. 360, 658-672 (2019)

66. Y.H. Yue, T.Y. Wang, M. Sakai, Y.S. Shen, Chem. Eng. Sci. 205, 121-133 (2019)

67. K. Wu, E.Q. Zhang, J. Xu, Z.L. Yuan, W.K. Zhu, B. Li, L. Wang, D.S. Luo, Int. J. Multiphase Flow 124, 103181 (2019)

68. C.M. Jia, Y.H. Dai, Y.H. Yang, J.W. Chew, Particuology 49, 55-64 (2019)

69. J. Li, Z.H. Luo, X.Y. Lan, C.M. Xu, J.S. Gao, Powder Technol. 237, 569-580 (2013)

70. T. Benjaprakairat, P. Piumsomboon, B. Chalermsinsuwan, Energy Rep. 6, 137-145 (2020)

71. D. Yan, H.Z. Li, Z. Zou, Q.S. Zhu, Comput. Appl. Chem. 34, 417-423 (2017)

72. Q.W. Liu, W.Q. Zhong, A.B. Yu, Powder Technol. 349, 40-51 (2019)

73. M. Tao (Harbin Institute of Technology, Harbin, 2019)

74. T. Zhou, S.L. Yang, Y.G. Wei, J.H. Hu, H. Wang, Renewable Energy 148, 534-537 (2019)

75. J. Xie, W.Q. Zhong, Y.J. Shao, K.X. Li, Powder Technol. 353, 72-83 (2019) 
76. J.X. Zheng, Y.L. Cong, J. Northeast Electr. Power Univ. 39, 41-48 (2019)

77. S. Jalalifar, M. Masoudi, R. Abbassi, V. Garaniya, M. Ghiji, F. Salehi, Energy 191, 1-12 (2019)

78. L.J. Xue, X.B. Li, Y.C. Wang, P.F. Shen, J. Chin. Soc. Power Eng. 9, 394-401 (2019)

79. L.R. Sun, K. Luo, J.R. Fan, J. Eng. Thermophys. 40, 1826-1830 (2019)

80. W.J. Yin (Harbin University of Science and Technology, Harbin, 2018)

81. L. Tian (Guizhou University, Guiyang, 2017)

82. S. Wang, W.J. Yin, S.Y. Liu, X.J. Song, Appl. Therm. Eng. 158, 113465 (2019)

83. T. Zhang (South China University of Technology, Guangzhou, 2012)

84. Z.Y. Duan, S.J. Sun, Z.J. Lan, Y. Wang, J.M. Zhang, J.T. Wang, Powder Technol. 372 428-437 (2020) 